\title{
Yield of Opportunistic Targeted Screening for Type 2 Diabetes in Primary Care: The Diabscreen Study
}

\author{
Erwin P. Klein Woolthuis, $M D^{1}$ \\ Wim J. C. de Grauw, MD, $P b D^{1}$ \\ Willem H. E. M. van Gerwen, MSc ${ }^{1}$ \\ Henk J. M. van den Hoogen, MSc ${ }^{1}$ \\ Eloy H. van de Lisdonk, $M D, P b D^{1}$ \\ Job F. M. Metsemakers, $M D, P b D^{2}$ \\ Chris van Weel, MD, PbD, FRCGP, \\ FRACGP ${ }^{1}$ \\ 'Department of Primary and Community \\ Care, Centre for Family Medicine, Geriatric \\ Care and Public Health, Radboud Univer- \\ sity Nijmegen Medical Centre, Nijmegen, \\ The Netherlands \\ ${ }^{2}$ Department of Family Medicine, \\ Maastricht University, Maastricht, \\ The Netherlands
}

AC

Annals Journal Club selection, see inside back cover or http://www annfammed.org/AJC/.

Conflicts of interest: none reported

\section{CORRESPONDING AUTHOR}

Erwin P. Klein Woolthuis, MD Radboud University Nijmegen Medical Centre

Department of Primary and Community Care, 117 HAG

PO Box 9101

6500 HB Nijmegen, The Netherlands

e.kleinwoolthuis@elg.umcn.nl

\begin{abstract}
PURPOSE In screening for type 2 diabetes, guidelines recommend targeting high-risk individuals. Our objectives were to assess the yield of opportunistic targeted screening for type 2 diabetes in primary care and to assess the diagnostic value of various risk factors.

METHODS In 11 family practices (total practice population $=49,229$ ) in The Netherlands, we conducted a stepwise opportunistic screening program among patients aged 45 to 75 years by (1) identifying high-risk individuals ( $\geq 1$ diabetes risk factor) and low-risk individuals using the electronic medical record, (2) obtaining a capillary fasting plasma glucose measurement, repeated on a separate day if the value was greater than $110 \mathrm{mg} / \mathrm{dL}$, and (3) obtaining a venous sample if both capillary fasting plasma glucose values were greater than $110 \mathrm{mg} / \mathrm{dL}$ and at least 1 sample was $126 \mathrm{mg} / \mathrm{dL}$ or greater. We calculated the yield (percentage of invited patients with undiagnosed diabetes), number needed to screen (NNS), and diagnostic value of the risk factors (odds ratio and area under the receiver operating characteristic curve).
\end{abstract}

RESULTS We invited for a first capillary measurement 3,724 high-risk patients seen during usual care and a random sample of 465 low-risk patients contacted by mail. The response rate was $90 \%$ and $86 \%$, respectively. Ultimately, 101 high-risk patients $(2.7 \%$; $95 \%$ confidence interval $[\mathrm{Cl}], 2.2 \%-3.3 \%$; NNS $=37)$ and 2 low-risk patients $(0.4 \% ; 95 \% \mathrm{Cl}, 0.1 \%-1.6 \%$; NNS $=233)$ had undiagnosed diabetes $(P<.01)$. The prevalence of diabetes among patients 45 to 75 years old increased from $6.1 \%$ to $6.8 \%$ as a result. Among diagnostic models containing various risk factors, a model containing obesity alone was the best predictor of undiagnosed diabetes (odds ratio $=3.2 ; 95 \% \mathrm{Cl}, 2.0-5.2$; area under the curve $=0.63$ ).

CONCLUSIONS The yield of opportunistic targeted screening was fair; obesity alone was the best predictor of undiagnosed diabetes. Opportunistic screening for type 2 diabetes in primary care could target middle-aged and older adults with obesity.

Ann Fam Med 2009;7:422-430. doi:10.1370/afm.997.

\section{INTRODUCTION}

$\mathrm{P}$ rimary care clinicians are encouraged to be more proactive in detecting and treating both diabetes and prediabetes. ${ }^{1}$ The recently updated standards of medical care of the American Diabetes Association (ADA) recommend testing adults of any age who are overweight or obese and have additional diabetes risk factors. ${ }^{2}$

The main reason to recommend screening for type 2 diabetes is the disease's long preclinical period of up to 12 years. The condition goes undiagnosed in one-third to one-half of all people with type 2 diabetes during this entire period, and they already have complications by the time of diagnosis. ${ }^{3}$ 
Starting treatment at an earlier stage might prevent or delay the development of diabetes complications. Studies have shown that in clinically detected (not screening-detected) diabetes, tight glycemic control can reduce the progression of microvascular disease, and that treatment of hypertension and hyperlipidemia decreases cardiovascular risk. ${ }^{4}$ Screening for and treating prediabetes - impaired fasting glucose (IFG) and impaired glucose tolerance-might prevent or slow the progression to diabetes and reduces the risk of cardiovascular disease. ${ }^{1,5}$

At present, however, randomized controlled trials have failed to show that earlier detection by screening reduces morbidity, mortality, or both among people with undiagnosed type 2 diabetes. ${ }^{4,6}$ There is also little knowledge about the ethical, psychological, and social consequences of screening results that are truly or falsely positive or negative, and there is no consensus on which screening tests to use and which diagnostic cutoff points are best. . $^{7-9}$

These considerations notwithstanding, screening for diabetes is encouraged nowadays. Targeting highrisk patients is recommended, as there is no evidence of a direct benefit of routine population-based screening for type 2 diabetes. ${ }^{2,4,10-12}$ As screening should also be a systematic and continuous process, ${ }^{7}$ opportunistic targeted screening might be a valuable screening method in primary care. This method entails screening high-risk individuals during usual care. ${ }^{6}$

The pragmatic nature of opportunistic targeted screening enables initiation of further diagnostic testing and treatment of newly diagnosed type 2 diabetes. To investigate this approach, we performed a study of a stepwise opportunistic screening program embedded in daily routine care in family practices in The Netherlands, targeting high-risk patients-the Diabscreen study. In the analysis reported here, our objectives were to assess the yield of our screening program and the diagnostic value of the risk factors we used in the study.

\section{METHODS}

\section{Participants and Setting}

Participants were recruited from 11 family practices in The Netherlands that were part of academic research networks of university departments of family medicine. The practices had a total population of 49,229 patients cared for by 25 family practitioners, and had not previously performed systematic screening for diabetes. Seven of the practices were from the Radboud University Nijmegen Medical Centre, ${ }_{1}^{13} 2$ were from Maastricht University, ${ }^{14}$ and 2 were from the Amsterdam VU University Medical Centre. ${ }^{15}$ We considered for inclusion in the study all patients aged 45 to 75 years inclusive who were listed with these practices and were not known to have diabetes. In The Netherlands, every individual in the population is registered with a family practitioner, usually the same one for many years. Patients need a referral by a family practitioner to consult a specialist.

All practices used an electronic medical record (EMR) with the same software (Promedico ICT Inc Nieuwegein, The Netherlands) containing relevant medical information, such as medical history, diagnoses, medication use, and referrals. Coding of diagnoses was based on the electronic version of the International Classification of Primary Care (ICPC) codes. ${ }^{16}$ Prescribed medication was coded according to Anatomical Therapeutic Chemical (ATC) codes. ${ }^{17}$

In our study, patients were defined as having undiagnosed type 2 diabetes if they had a venous fasting plasma glucose (FPG) value of at least $126 \mathrm{mg} / \mathrm{dL}$ ** They were defined as having IFG if they had a venous FPG value of greater than $110 \mathrm{mg} / \mathrm{dL}$ and less than $126 \mathrm{mg} / \mathrm{dL} .^{18,19} \mathrm{We}$ did not study impaired glucose tolerance, because our pragmatic screening protocol involved only FPG testing and not an oral glucose challenge.

Ethical approval for the study was obtained from the Radboud University Nijmegen Medical Centre ethics committee.

\section{Screening Program}

Our opportunistic screening program consisted of a stepwise screening procedure: (1) using the EMR, identification of high-risk and low-risk individuals; (2) a first capillary FPG measurement and, if indicated by the result, a second one; and (3) if indicated by that result, a venous FPG.

We considered patients to be at high risk for undiagnosed type 2 diabetes if they had 1 or more of the following diabetes risk factors, derived from the ADA recommendations for screening for type 2 diabetes $^{2}$ : a family history of diabetes (defined as diabetes in a parent, brother, or sister, or some combination thereof), hypertension, cardiovascular disease (myocardial infarction, heart failure, atrial fibrillation, stroke, peripheral vascular disease), lipid metabolism disorders, obesity (body mass index $>27 \mathrm{~kg} / \mathrm{m}^{2}$ ), and a history of gestational diabetes mellitus. ${ }^{2,11}$

We translated these risk factors into a set of matching ICPC and ATC codes. ${ }^{20}$ Using a computerized cross-sectional analysis of ICPC and ATC information for each patient from the practices' EMR, we determined the patients' diabetes risk status (high vs

* Conversion factor to SI units is $\times 0.0555$. 
low) and entered it in the EMR. During a usual care consultation in the following year, the EMR reminded the family practitioners to verify and, in the case of missing data, complete the patients' risk profile and to invite high-risk patients for an FPG measurement. As part of daily practice, an appointment for this test was recorded in the practice schedule. There were no further reminders.

In addition, to assess the yield of opportunistic screening among low-risk patients, from each participating practice, we also invited for FPG measurement a random sample of low-risk patients: patients from the same age-group, but without any of the risk factors listed above. On the basis of an expected prevalence of undiagnosed type 2 diabetes of $0.5 \%,{ }^{21}$ an intraclass correlation coefficient of 0.03 , and a desired precision of $1 \%$, we calculated a required sample size of 380 lowrisk patients. These patients were randomly selected from a list of low-risk patients and subsequently invited by mail to visit the practice for screening.

Our stepwise screening protocol was based on cutoff points used for IFG and diabetes. ${ }^{22}$ All patients with an initial capillary FPG of greater than $110 \mathrm{mg} / \mathrm{dL}$ were invited for a second capillary measurement on another day. This second measurement was immediately followed by a venous FPG measurement if both capillary measurements were greater than $110 \mathrm{mg} / \mathrm{dL}$ and at least 1 was $126 \mathrm{mg} / \mathrm{dL}$ or greater.

\section{Measurements}

Measurements were made in the patients' own family practice by the regular practice assistants. Capillary samples were taken using a Gluco Touch plasma-calibrated capillary blood glucose meter (LifeScan Benelux, Beerse, Belgium). Before the start of the study, all participating practices received new meters, which were checked and adjusted, if necessary, by the manufacturer. The practice assistants were trained in using the meters. Venous samples were sent to a central laboratory for further analysis in a Roche/Hitachi chemical analyzer (Roche Nederland BV, Woerden, The Netherlands), using the glucose oxidase method.

\section{Data Analysis}

We analyzed data with SPSS 16.0 for Windows (SPSS Inc, Chicago, Illinois). Statistical analysis was performed using the $\chi^{2}$ test for categorical data and the Student $t$ test or Kruskal-Wallis test for means where appropriate. We considered a $P$ value $<.05$ to be significant.

We calculated the yield of our screening program (the percentage of invited patients found to have undiagnosed type 2 diabetes); the number of patients who would need to be invited for screening in order to identify 1 patient with undiagnosed type 2 diabetes, or number needed to screen (NNS); and the change in diabetes prevalence among the study population resulting from the program.

We examined possible interactions between the risk factors by calculating the correlation coefficients Then, we quantified their association with the presence or absence of undiagnosed diabetes using univariate logistic regression analysis. Variables with a $P$ value $\leq .15$ were included in multivariate binary logistic regression analysis to determine their independent contribution to the risk of undiagnosed type 2 diabetes. Using the backward stepwise (likelihood ratio) method, excluding variables one by one, we were able to produce diagnostic models with an area under the receiver operating characteristic curve (AUC).

\section{RESULTS}

The 11 participating practices had 49,229 registered patients (2,500-9,750 per practice), of whom 14,457 (957-1,831 per practice) were aged 45 to 75 years (Figure 1). The prevalence of known diabetes before our screening program was $6.1 \%$, leaving 13,581 patients for the study. During the 1-year study period, 5,277 (39\%) of these patients had an encounter with a family practitioner during which screening was discussed. Risk assessment indicated that 3,724 (71\%) were at high risk for diabetes and 1,553 (29\%) were at low risk $; 90 \%$ of the high-risk patients and $86 \%$ of the 465 invited low-risk patients returned for a first capillary FPG measurement after invitation. Sex and mean age did not differ significantly between high-risk and lowrisk patients, but mean FPG was slightly higher in the former group (Table 1).

\section{High-Risk Patients}

A second capillary FPG was performed in 496 high-risk patients, or $88 \%$ of those invited (Figure 1). According to our protocol, 169 (5\%) were eligible for venous FPG measurement immediately after the second capillary FPG measurement. A venous sample was collected in $125(74 \%)$ of these patients but not in $44(26 \%)$. In the latter group, the second capillary FPG more often was 110 to $126 \mathrm{mg} / \mathrm{dL}$, but other characteristics did not differ significantly between groups (Table 2). Of the 125 patients with a venous sample, $81 \%$ had undiagnosed type 2 diabetes, $16 \%$ had IFG, and $3 \%$ had a normal fasting glucose level. These groups differed significantly in terms of mean FPG values and the prevalence of lipid metabolism disorders (Table 3).

\section{Low-Risk Patients}

In the low-risk group, only 2 patients had undiagnosed type 2 diabetes and 1 patient had IFG (Figure 1). The 


\section{Figure 1. Study population and algorithm of the screening procedure.}

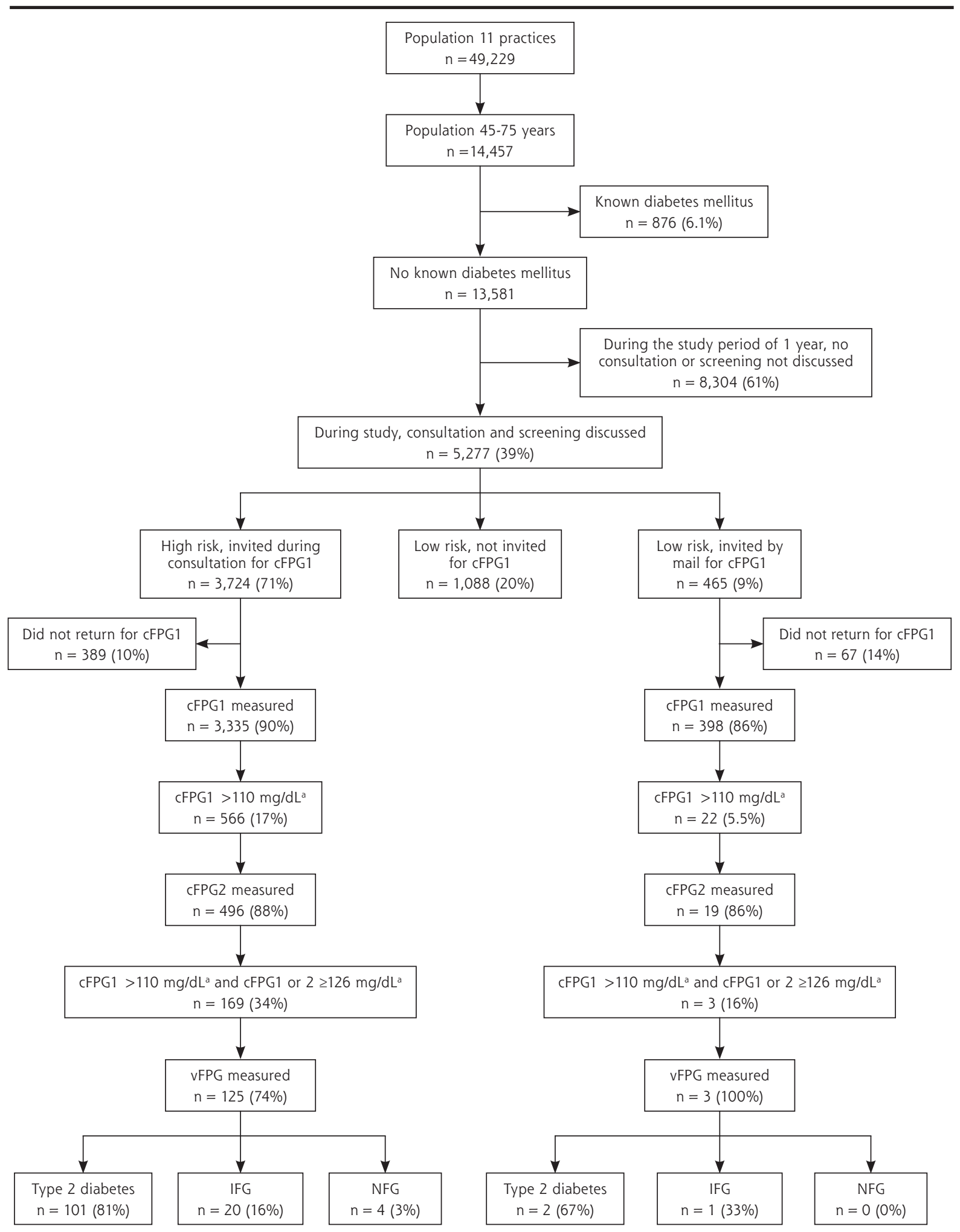

cFPG1 = first capillary fasting plasma glucose; cFPG2 = second capillary fasting plasma glucose; $v F P G$ = venous fasting plasma glucose; NFG = normal fasting plasma glucose; IFG = impaired fasting glucose.

a Conversion factor to SI units: $\times 0.0555$. 
characteristics within each subgroup are displayed in Table 3. Further analysis was not possible in this group because of the small number of patients.
Yield of the Screening Program

We found undiagnosed type 2 diabetes in 101 highrisk patients and 2 low-risk patients. These values
Table 1. Baseline Characteristics of High-Risk and Low-Risk Patients in Whom a First Capillary Fasting Plasma Glucose Level Was Measured

\begin{tabular}{|c|c|c|c|}
\hline Characteristic & $\begin{array}{c}\text { High-Risk } \\
\text { Patients } \\
(n=3,335)\end{array}$ & $\begin{array}{c}\text { Low-Risk } \\
\text { Patients } \\
(n=398)\end{array}$ & $P$ Value \\
\hline Sex (male), No. (\%) & $1,411(42.3)$ & $168(42.2)$ & .97 \\
\hline Age, mean (SD), years & $58.2(8.2)$ & $57.5(7.2)$ & .07 \\
\hline \multicolumn{4}{|l|}{ cFPG1 } \\
\hline cFPG1, mean (SD), mg/dLa & $99.1(21.6)$ & $93.7(10.8)$ & $<.001$ \\
\hline cFPG1 110-126 mg/dL, No. (\%) & $394(11.8)$ & $16(4.0)$ & $<.001$ \\
\hline cFPG1 $\geq 126$ mg/dL, No. (\%) & $172(5.2)$ & $6(1.5)$ & $<.001$ \\
\hline \multicolumn{4}{|l|}{$\mathrm{BMI}^{\mathrm{b}}$} \\
\hline BMI, mean (SD), $\mathrm{kg} / \mathrm{m}^{2}$ & $28.0(4.5)$ & $23.5(2.2)$ & $<.001$ \\
\hline $\mathrm{BMI}>27$ kg/m², No. (\%) & $1,786(57.4)$ & 0 & - \\
\hline \multicolumn{4}{|l|}{ Risk factors } \\
\hline Hypertension, No. (\%) & $814(24.4)$ & 0 & - \\
\hline Cardiovascular disease, No. (\%) & $499(15.0)$ & 0 & - \\
\hline Lipid metabolism disorders, ${ }^{c}$ No. (\%) & $319(32.1)$ & 0 & - \\
\hline Family history of diabetes, No. (\%) & $1,288(38.6)$ & 0 & - \\
\hline History of GDM, ${ }^{d}$ No. (\%) & $17(2.8)$ & 0 & - \\
\hline $\begin{array}{l}\text { BMI = body mass index; } \text { cFPG1 = first capillary } \\
\text { a Conversion factor to SI units: } \times 0.0555 \text {. } \\
\text { b Missing }=225 \text {. } \\
{ }^{\mathrm{C}} \text { Missing }=2,342 \text {. } \\
{ }^{\mathrm{d}} \text { Missing }=2,726 \text {. }\end{array}$ & asting plasma glucc & GDM = gestation & abetes mellit \\
\hline
\end{tabular}

corresponded to $2.7 \%$ of high-risk patients (95\% confidence interval [CI], 2.2\%-3.3\%; NNS = 37) vs $0.4 \%$ of low-risk patients $(95 \% \mathrm{CI}$, $0.1 \%-1.6 \%$; NNS = 233) invited for screening $(P<.01)$. As a result of the screening program, the prevalence of known diabetes among patients aged 45 to 75 years in the study practices increased from $6.1 \%(876$ patients) to $6.8 \%$ (979 patients).

\section{Diagnostic Value of the Risk Factors}

There were significant but no relevant correlations between the risk factors. For example, the Spearman rank correlation coefficient of obesity with hypertension was 0.08 with $P<.01$ (data not further shown).

Univariate logistic regression analysis showed that the odds of undiagnosed type 2 diabetes were significantly higher among patients who were obese (odds ratio $[\mathrm{OR}]=3.2 ; 95 \% \mathrm{CI}, 2.0-5.2)$ or had

Table 2. Characteristics of High-Risk Patients Eligible For Venous FPG Measurement, Comparing Patients With and Without a Venous Sample

\begin{tabular}{|c|c|c|c|}
\hline Characteristic & $\begin{array}{c}\text { Patients With } \\
\text { Venous Sample } \\
(n=125)\end{array}$ & $\begin{array}{l}\text { Patients Without } \\
\text { Venous Sample } \\
\qquad(n=44)\end{array}$ & $P$ Value \\
\hline Sex (male), No. (\%) & $57(45.6)$ & $23(52.3)$ & .45 \\
\hline Age, mean (SD), years & $58.8(8.0)$ & $58.5(8.1)$ & .87 \\
\hline \multicolumn{4}{|l|}{ cFPG measurements } \\
\hline cFPG1, mean (SD), mg/dLa & $156.8(55.9)$ & $151.4(50.5)$ & .59 \\
\hline CFPG2, mean (SD), mg/dL & $149.5(41.4)$ & $140.5(37.8)$ & .23 \\
\hline CFPG1 $110-126 \mathrm{mg} / \mathrm{dL}$ and $\mathrm{CFPG} 2 \geq 126 \mathrm{mg} / \mathrm{dL}$, No. (\%) & $28(22.4)$ & $6(13.6)$ & .21 \\
\hline cFPG1 $\geq 126 \mathrm{mg} / \mathrm{dL}$ and $\mathrm{CFPG} 2110-126 \mathrm{mg} / \mathrm{dL}$, No. (\%) & $22(17.6)$ & $21(47.8)$ & $<.001$ \\
\hline cFPG1 $\geq 126 \mathrm{mg} / \mathrm{dL}$ and $\mathrm{cFPG} 2 \geq 126 \mathrm{mg} / \mathrm{dL}$, No. (\%) & $75(60.0)$ & $17(38.6)$ & .01 \\
\hline BMI, mean (SD), kg/m² & $30.2(4.6)$ & $31.0(6.9)$ & .39 \\
\hline \multicolumn{4}{|l|}{ Risk factors } \\
\hline Hypertension, No. (\%) & $49(39.2)$ & $15(34.1)$ & .55 \\
\hline Cardiovascular disease, No. (\%) & $22(17.6)$ & $5(11.4)$ & .33 \\
\hline Lipid metabolism disorders, ${ }^{\mathrm{b}}$ No. (\%) & $12(38.7)$ & $3(21.4)$ & .41 \\
\hline Family history of diabetes, No. (\%) & $58(46.4)$ & $17(38.6)$ & .37 \\
\hline History of GDM, ${ }^{c}$ No. (\%) & 0 & 0 & - \\
\hline \multicolumn{4}{|c|}{$\begin{array}{l}\mathrm{BMI}=\text { body mass index; FPG = fasting plasma glucose; } \mathrm{CFPG1}=\text { first capillary fasting plasma glucose; } \mathrm{CFPG} 2=\text { second capillary fasting plasma glucose; } G D M=\text { gesta- } \\
\text { tional diabetes mellitus. }\end{array}$} \\
\hline \multicolumn{4}{|l|}{ a Conversion factor to SI units: $\times 0.0555$. } \\
\hline \multicolumn{4}{|l|}{$\begin{array}{l}{ }^{b} \text { Missing }=94 \text { with venous sample; } 30 \text { without venous sample. } \\
{ }^{c} \text { Missing }=100 \text { with venous sample; } 26 \text { without venous sample. }\end{array}$} \\
\hline
\end{tabular}


Table 3. Characteristics of High-Risk and Low-Risk Patients in Venous FPG Subgroups

\begin{tabular}{|c|c|c|c|c|c|c|}
\hline \multirow[b]{2}{*}{ Characteristic } & \multicolumn{3}{|c|}{ High-Risk Patients } & \multicolumn{3}{|c|}{ Low-Risk Patients } \\
\hline & $\begin{array}{l}\text { Diabetes } \\
(\mathrm{n}=101)\end{array}$ & $\begin{array}{c}\text { IFG } \\
(n=20)\end{array}$ & $\begin{array}{c}\text { NFG } \\
(n=4)\end{array}$ & $\begin{array}{c}\text { Diabetes } \\
(n=2)\end{array}$ & $\begin{array}{l}\text { IFG } \\
(n=1)\end{array}$ & $\begin{array}{l}\text { NFG } \\
(n=0)\end{array}$ \\
\hline Sex (male), No. (\%) & $49(48.5)$ & $6(30.0)$ & $2(50.0)$ & $1(50.0)$ & 0 & 0 \\
\hline Age, mean (SD), years & $59.4(8.1)$ & $56.0(7.8)$ & $55.8(2.6)$ & $67.5(6.4)$ & 55.0 & 0 \\
\hline \multicolumn{7}{|l|}{ Plasma glucose levela } \\
\hline cFPG1, mean (SD), mg/dL & $162.2(59.5)$ & $129.7(12.6)$ & $126.0(7.2)^{b}$ & $127.9(1.8)$ & 127.9 & 0 \\
\hline cFPG2, mean (SD), mg/dL & $155.0(43.2)$ & $124.3(14.4)$ & $117.1(7.2)^{c}$ & $135.1(3.6)$ & 129.7 & 0 \\
\hline vFPG, mean (SD), mg/dL & $164.0(41.4)$ & $120.7(3.6)$ & $104.5(5.4)^{d}$ & $133.3(7.2)$ & 110.0 & 0 \\
\hline \multicolumn{7}{|l|}{$\mathrm{BMl}^{\mathrm{e}}$} \\
\hline $\mathrm{BMI}$, mean (SD), kg/m² & $29.9(3.9)$ & $32.2(6.9)$ & $28.2(4.8)$ & $25.3(1.5)$ & 25.3 & 0 \\
\hline BMI > 27 kg/m², No. (\%) & $73(78.5)$ & $17(89.5)$ & $2(50.0)$ & 0 & 0 & 0 \\
\hline \multicolumn{7}{|l|}{ Risk factors } \\
\hline Hypertension, No. (\%) & $41(40.6)$ & $7(35.0)$ & $1(25.0)$ & 0 & 0 & 0 \\
\hline Cardiovascular disease, No. (\%) & $17(16.8)$ & $5(25.0)$ & 0 & 0 & 0 & 0 \\
\hline Lipid metabolism disorders, ${ }^{\dagger}$ No. (\%) & $6(23.1)$ & $6(66.7)$ & $0^{\mathrm{b}}$ & 0 & 0 & 0 \\
\hline Family history of diabetes, No. (\%) & $43(42.6)$ & $13(65.0)$ & $2(50.0)$ & 0 & 0 & 0 \\
\hline History of GDM, ${ }^{9}$ No. (\%) & 0 & 0 & 0 & 0 & 0 & 0 \\
\hline \multicolumn{7}{|c|}{$\begin{array}{l}\mathrm{BMI}=\text { body mass index } ; \mathrm{CFPG1}=\text { first capillary fasting plasma glucose; } \mathrm{CFPG2}=\text { second capillary fasting plasma glucose; } F \mathrm{FG}=\text { fasting plasma glucose; } \mathrm{vFPG}=\text { venou } \\
\text { fasting plasma glucose; } \mathrm{GDM}=\text { gestational diabetes mellitus; } \mathrm{IFG}=\text { impaired fasting glucose; } \mathrm{NFG}=\text { normal fasting glucose. }\end{array}$} \\
\hline \multicolumn{7}{|c|}{ Note: Statistical analysis of the low-risk group was not possible because of the small numbers. } \\
\hline $\begin{array}{l}\text { a Conversion factor to SI units: } \times 0.0555 \text {. } \\
\text { b } P<.05 \text { in high-risk group. } \\
\text { ' } P<.01 \text { in high-risk group. } \\
\text { d } P<.001 \text { in high-risk group. } \\
\text { e Missing in high-risk group }=8 \text { with diabetes } \\
\text { ' Missing in high-risk group }=75 \text { with diabete } \\
{ }^{\circ} \text { Missing in high-risk group }=80 \text { with diabete }\end{array}$ & $\begin{array}{l}\text { IFG; } 0 \text { with NFG } \\
\text { th IFG; } 3 \text { with NF } \\
\text { ith IFG; } 3 \text { with N }\end{array}$ & & & & & \\
\hline
\end{tabular}

hypertension $(\mathrm{OR}=2.5 ; 95 \% \mathrm{CI}, 1.6-3.8)$ (Table 4). In contrast, a family history of diabetes was not significantly associated with undiagnosed diabetes $(\mathrm{OR}=$ $1.4 ; 95 \% \mathrm{CI}, 0.9-2.1)$. Because of the large number of missing data, lipid metabolism disorders and history of gestational diabetes mellitus were not included in the analysis.

Multivariate binary logistic regression analysis showed that obesity was the best predictor of undiagnosed type 2 diabetes: $76.8 \%$ of those with the disease

Table 4. Univariate Analysis of the Association Between Diabetes Risk Factors and the Odds of Undiagnosed Type 2 Diabetes

\begin{tabular}{|c|c|c|c|c|}
\hline \multirow[b]{2}{*}{ Risk Factor } & \multicolumn{2}{|c|}{ Undiagnosed Diabetes } & \multirow[b]{2}{*}{$\begin{array}{c}\text { Odds Ratio } \\
\text { (95\% Cl) }\end{array}$} & \multirow[b]{2}{*}{$P$ Value } \\
\hline & $\begin{array}{l}\text { Yes, No. (\%) } \\
(n=95)\end{array}$ & $\begin{array}{l}\text { No, No. (\%) } \\
(\mathrm{n}=3,379)\end{array}$ & & \\
\hline Sex (male) & $46(48.4)$ & $1,431(42.3)$ & $1.3(0.9-1.9)$ & .24 \\
\hline Age $>60$ years & $45(47.4)$ & $1,406(41.6)$ & $1.3(0.8-1.9)$ & .26 \\
\hline Hypertension & $37(38.9)$ & $691(20.4)$ & $2.5(1.6-3.8)$ & $<.001$ \\
\hline Cardiovascular disease & $16(16.8)$ & $429(12.7)$ & $1.4(0.8-2.4)$ & .23 \\
\hline Obesity (BMI > 27 kg/m²) & $73(76.8)$ & $1,713(50.7)$ & $3.2(2.0-5.2)$ & $<.001$ \\
\hline Family history of diabetes & $41(43.2)$ & $1,212(35.9)$ & $1.4(0.9-2.1)$ & .15 \\
\hline
\end{tabular}

were obese (AUC $=0.63 ; 95 \%$ confidence interval, 0.58-0.68) (Table 5). Hypertension and family history of diabetes were poorer predictors.

\section{DISCUSSION}

The yield of our opportunistic targeted screening program was fair: in 1 year we identified undiagnosed type 2 diabetes in 101 high-risk patients invited for screening $(2.7 \%$, NNS $=37)$. This number represents $30 \%$ of cases of known diabetes, considering that $39 \%$ of the study population had an encounter with a family practitioner (39\% of 876 patients previously known to have diabetes $=342$, and $101 / 342=30 \%)$. The yield of screening in low-risk patients was, as expected, only $0.4 \%$ (NNS = 233). The response rate for the capillary measurements was high, at about $90 \%$. As a result of the screening program, the prevalence of known diabetes among our patients aged 45 to 75 years 


\begin{tabular}{|c|c|c|c|c|}
\hline Model & $\begin{array}{c}\text { Odds Ratio } \\
(95 \% \mathrm{Cl})\end{array}$ & $\begin{array}{c}P \\
\text { Value }\end{array}$ & $\begin{array}{l}\text { Undiagnosed } \\
\text { Diabetes, } \\
\text { No. }(\%) \\
(n=95)\end{array}$ & AUC $(95 \% \mathrm{Cl})^{\mathrm{a}}$ \\
\hline Model 1 & & & $12(12.6)$ & $0.54(0.48-0.61)$ \\
\hline Obesity (BMI $>27 \mathrm{~kg} / \mathrm{m}^{2}$ ) & $3.1(1.9-5.0)$ & $<.001$ & - & - \\
\hline Hypertension & $2.3(1.5-3.5)$ & $<.001$ & - & - \\
\hline Family history of diabetes & $1.4(1.0-2.2)$ & .09 & - & - \\
\hline Model 2 & & & $30(31.6)$ & $0.60(0.54-0.66)$ \\
\hline Obesity & $3.0(1.9-4.9)$ & $<.001$ & - & - \\
\hline Hypertension & $2.3(1.5-3.5)$ & $<.001$ & - & - \\
\hline Model 3 & & & $73(76.8)$ & $0.63(0.58-0.68)$ \\
\hline Obesity & $3.2(2.0-5.2)$ & $<.001$ & - & - \\
\hline
\end{tabular}

$\mathrm{AUC}=$ area under the receiver operating characteristic curve; $\mathrm{BMI}=$ body mass index; $\mathrm{Cl}=$ confidence interval. Note: Only risk factors with $P \leq .15$ in Table 4 were included.

a An AUC of 0.50 means that the model does not predict the outcome better (more accurately) or worse (less accurately) than random guess; an AUC greater than 0.50 means that the prediction is better than random, and an AUC less than 0.50 means that the prediction is worse than random.

test consists of an FPG measurement plus a 2-hour plasma glucose measurement, and has been considered to be the criterion standard in diagnosing diabetes The FPG test is, nevertheless, recommended for screening in primary care as it is easier and faster to perform, more convenient and acceptable to patients, and less expensive. ${ }^{2,24}$

Our focus was on testing the applicability of our stepwise protocol during usual care; therefore, we did not collect 3 blood samples (2 capillary and 1 venous) from all study participants. With an $81 \%$ concordance between identification for venous sampling and an undiagnosed type 2 diabetes outcome in high-risk patients (positive

increased from $6.1 \%$ to $6.8 \%$. Of the ADA diabetes risk factors, obesity was the best predictor of undiagnosed type 2 diabetes.

The main strength of the study was the setting. High-risk patients were invited for screening during daily routine practice in the patients' local family practice by their own family practitioner. Capillary blood samples were taken by the practice assistants, without any further support (eg, from trial nurses). Although patients had to return in a fasting state for the capillary FPG measurements, they were highly willing to do so. And although all participating family practices were related to a university department of family medicine, they were standard community practices with a population representative of the Dutch population and a diabetes prevalence equal to that in The Netherlands. ${ }^{13-15,23}$ Because the Dutch system of primary health care provides for universal access and continuity of patient registration, we were able to use the family practice EMR in a continuous screening program.

Our screening approach calls for the identification of individual risk factors during a regular consultation. To the extent possible, we used available information from the EMR, which is based on the ICPC. A limiting factor is that not all risk factors are included in the ICPC at this time; therefore, we had to ask patients about their risk factors to confirm their status. ${ }^{20}$ Our study supports the relevance of routine inclusion of risk factors in the EMR and the importance of expanding the ICPC to include this information.

A possible limitation was that we used the FPG test rather than the oral glucose tolerance test. The latter predictive value $=81 \%$, our protocol was very useful Since this protocol was designed to screen for undiagnosed type 2 diabetes, few patients who underwent venous sampling were found to have IFG.

In $26 \%$ of our high-risk patients eligible for a venous sample, this measurement was not performed. Considering the high level of compliance with the capillary measurements, the general lack of significant differences between patients with a venous measurement and those without, and the requirement by the protocol that the venous sample be obtained immediately after the second capillary measurement, we believe that the missing venous samples were mainly due to protocol factors (eg, misinterpretation-willingly or not-by the practice assistants) instead of patient factors. The fact that high-risk patients without a venous sample more often had a second capillary FPG of 110 to $126 \mathrm{mg} / \mathrm{dL}$ supports this assumption. Instead of giving assistants a flowchart with all possible combinations of glucose outcomes (not described) as we did in the study, we now believe it would have been more helpful if we had given them the simple algorithm mentioned in the "Methods" section. We estimate that if compliance had been $100 \%$, the number of newly diagnosed cases of diabetes among high-risk patients could in fact have been 136, giving an even lower NNS of 28.

The portable glucose meters we used are userfriendly and readily available in primary care. A potential drawback is their variability, ${ }^{25}$ and consequent risk of false-positive and false-negative outcomes. Our stepwise approach, in which patients with glucose levels above the threshold underwent measurement again, 
did address the problems of false-positive results. ${ }^{22}$ To address false-negative results, the procedure must be repeated, for example, every 3 years, as recommended by the ADA. ${ }^{2}$

Since we wanted to perform a screening program embedded in daily care without any further support, we did not specifically study disadvantages or harms, or cost-effectiveness of our opportunistic screening program, nor did we specifically investigate acceptability of the screening procedure. As the program was embedded in daily care and the patient attendance rate was $90 \%$, however, we believe we can conclude that it was inexpensive and feasible. Further research is needed, though.

Several diabetes screening studies have been described in the literature. Smith et $\mathrm{al}^{26}$ undertook an opportunistic diabetes screening study performed in family practice using a questionnaire presented to patients who were waiting to see their doctor. Their participation rate was also high (93\%), and $43 \%$ of patients had at least 2 risk factors. If performed continuously or repeated regularly, such an approach might provide more complete and up-to-date information on a patient's risk status in the EMR, improving the identification of high-risk patients for screening purposes.

Greaves et $\mathrm{al}^{27}$ showed that identifying patients with type 2 diabetes and IFG using data stored in family practice databases was feasible (NNS $=21-38$ for type 2 diabetes), but instead of using an opportunistic approach, they invited high-risk patients (those aged $>50$ years and with a body mass index $\geq 27 \mathrm{~kg} / \mathrm{m}^{2}$ ) to screening clinics run by trained practice nurses. The response rate was $61 \%$. Nevertheless, the simple screening system they describe-like ours-would promote efficient use of scarce primary health care resources, especially when set up as part of a broader screening program to reduce cardiovascular disease.

In a cross-sectional study in a local family practice, Lawrence et $\mathrm{al}^{21}$ showed that screening of invited patients whose sole risk factor for diabetes is age older than 45 years has a low yield. In this group, they found a diabetes prevalence of just $0.2 \%$. Among individuals with 1 or more other risk factors, the figure increased to $2.8 \%$. Both prevalences are comparable to ours.

Recently, a population-based screening program for type 2 diabetes was performed in The Netherlands. ${ }^{12}$ Although the increase in diabetes prevalence achieved with the program (from $6.1 \%$ to $7.0 \%$ among people aged 50 to 70 years) was comparable to ours, the response to an invitation to glucose testing was $31 \%$ and the yield was only $1 \%$. The authors concluded that opportunistic screening might be more appropriate.

Primary care practices often have large patient populations, underscoring the need for a targeted approach to screening. In our family practices, more than twothirds of middle-aged and older study patients eligible for screening were at high risk. But largely because of the stepwise protocol, the yield of our opportunistic targeted screening method was fair.

In a recently updated statement, ${ }^{28}$ the US Preventive Services Task Force recommends screening for type 2 diabetes in asymptomatic adults with hypertension. As part of an assessment of cardiovascular disease risk, clinicians should also screen for diabetes to adequately assess patients' risk for this condition as well. ${ }^{28}$ With ever greater integration of diabetes screening into cardiovascular risk management, opportunistic screening for type 2 diabetes in primary care could target middle-aged and older adults with obesity. With this approach, the number of high-risk patients to be screened would be considerably reduced. A similar approach was found to be cost-effective. ${ }^{29}$

With an opportunistic targeted screening program like ours, diabetes screening in primary care can be performed systematically and continuously, with probably few drawbacks for both patients and health care workers, and with efficient use of resources. Further research is needed to estimate its cost-effectiveness and limitations. Also, sensitivity and specificity of our stepwise approach need to be studied.

In conclusion, the yield of opportunistic targeted screening in our study was fair, and obesity alone was the best predictor of undiagnosed diabetes. Our data confirm a low yield when low-risk individuals are screened. As diabetes screening is increasingly integrated into cardiovascular risk management, opportunistic screening for type 2 diabetes in primary care could target middle-aged and older adults with obesity.

To read or post commentaries in response to this article, see it online at http://www.annfammed.org/cgi/content/full/7/5/422.

Key words: Diabetes; diagnosis; family practice; practice-based research; primary health care; risk factors; screening

Submitted May 1, 2008; submitted, revised, December 7, 2008; accepted January 5, 2009.

Funding support: This study was funded by the Netherlands Organisation for Health Research and Development (ZonMw) and supported by Lifescan, who unconditionally provided the blood glucose meters.

Acknowledgments: We thank all participating family practitioners, practice assistants, and patients for their cooperation. We also thank Heert Tigchelaar for his work on the initial study design and data collection.

\section{References}

1. Kenealy T, Elley CR, Arroll B. Screening for diabetes and prediabetes. Lancet. 2007;370(9603):1888-1889.

2. American Diabetes Association. Standards of medical care in diabetes-2008. Diabetes Care. 2008;31(Suppl 1):S12-S54. 
3. Cowie CC, Harris MI, Eberhardt MS. Frequency and determinants of screening for diabetes in the U.S. Diabetes Care. 1994;17(10): 1158-1163.

4. Norris SL, Kansagara D, Bougatsos C, Fu R; US Preventive Services Task Force. Screening adults for type 2 diabetes: a review of the evidence for the US Preventive Services Task Force. Ann Intern Med. 2008;148(11):855-868.

5. Waugh N, Scotland G, McNamee $P$, et al. Screening for type 2 diabetes: literature review and economic modelling. Health Technol Assess. 2007;11(7):iii-xi, 1-125.

6. World Health Organization. Screening for Type 2 Diabetes. Report of a World Health Organization and International Diabetes Federation meeting. Geneva, Switzerland: World Health Organization, 2003. http://www.who.int/diabetes/publications/en/. Accessed 7 Dec 2008

7. Engelgau MM, Narayan KM, Herman WH. Screening for type 2 diabetes. Diabetes Care. 2000;23(10):1563-1580.

8. Wareham NJ, Griffin SJ. Should we screen for type 2 diabetes? Evaluation against National Screening Committee criteria. BMJ. 2001;322(7292):986-988.

9. Adriaanse MC, Snoek FJ, Dekker JM, et al. No substantial psychological impact of the diagnosis of type 2 diabetes following targeted population screening: The Hoorn Screening Study. Diabet Med. 2004;21(9):992-998.

10. Diabetes UK. Early Identification of People with Type 2 Diabetes: Position Statement. London, England: Diabetes UK, 2006. http://www. diabetes.org.uk. Accessed 7 Dec 2008.

11. Bouma M, Rutten GE, de Grauw WJ, Wiersma T, Goudswaard AN. [Summary of the practice guideline 'Diabetes mellitus type 2' (second revision) from the Dutch College of General Practitioners] [Article in Dutch; English abstract] Ned Tijdschr Geneeskd. 2006; 150(41):2251-2256.

12. Janssen PG, Gorter KJ, Stolk RP, Rutten GE. Low yield of population-based screening for type 2 diabetes in The Netherlands: the ADDITION Netherlands study. Fam Pract. 2007;24(6):555-561.

13. van Weel C. Longitudinal research and data collection in primary care. Ann Fam Med. 2005;3(Suppl 1):S46-S51.

14. Metsemakers JF, Höppener P, Knottnerus JA, Kocken RJ, Limonard CB. Computerized health information in The Netherlands: a registration network of family practices. Br J Gen Pract. 1992;42(356): 102-106.

15. Renders CM, Valk GD, Franse LV, Schellevis FG, van Eijk JT, van der Wal G. Long-term effectiveness of a quality improvement program for patients with type 2 diabetes in general practice. Diabetes Care. 2001;24(8):1365-1370.

16. Lamberts H, Wood M. ICPC. International Classification of Primary Care. Oxford, England: Oxford University Press; 1987
17. Guidelines for ATC Classification and DDD Assignment. Oslo, Norway: WHO Collaborating Centre for Drug Statistics Methodology, 2003. http://www.whocc.no/atcddd/. Accessed 7 Dec 2008.

18. The Expert Committee on the Diagnosis and Classification of Diabetes Mellitus. Report of the Expert Committee on the Diagnosis and Classification of Diabetes Mellitus. Diabetes Care. 1997;20(7):1183-1197.

19. World Health Organization. Definition and Diagnosis of Diabetes Mellitus and Intermediate Hyperglycemia: Report of a WHO/IDF Consultation. 1: Diabetes Mellitus-Diagnosis. Geneva, Switzerland: World Health Organization, 2006. http://www.who.int/diabetes/publications/en/. Accessed 7 Dec 2008.

20. Klein Woolthuis EP, de Grauw WJ, van Gerwen WH, et al. Identifying people at risk for undiagnosed type 2 diabetes using the GP's electronic medical record. Fam Pract. 2007;24(3):230-236.

21. Lawrence JM, Bennett $P$, Young A, Robinson AM. Screening for diabetes in general practice: cross sectional population study. BMJ. 2001;323(7312):548-551

22. Klein Woolthuis EP, de Grauw WJ, van Gerwen WH, et al. Screening for type 2 diabetes in primary care using a stepwise protocol: the Diabscreen study. Prim Care Diabetes. 2007;1(4):199-202.

23. Fleming DM, Schellevis FG, Van Casteren V. The prevalence of known diabetes in eight European countries. Eur J Public Health. 2004;14(1):10-14

24. Genuth S, Alberti KG, Bennett $P$, et al; Expert Committee on the Diagnosis and Classification of Diabetes Mellitus. Follow-up report on the diagnosis of diabetes mellitus. Diabetes Care. 2003;26(11): 3160-3167.

25. Colagiuri S, Sandbaek A, Carstensen B, et al. Comparability of venous and capillary glucose measurements in blood. Diabet Med. 2003;20(11):953-956.

26. Smith SM, Holohan J, McAuliffe A, Firth RG. Irish diabetes detection programme in general practice. Diabet Med. 2003;20(9):717-722.

27. Greaves CJ, Stead JW, Hattersley AT, Ewings P, Brown P, Evans PH A simple pragmatic system for detecting new cases of type 2 diabetes and impaired fasting glycaemia in primary care. Fam Pract. 2004;21(1):57-62.

28. U.S. Preventive Services Task Force. Screening for type 2 diabetes mellitus in adults: U.S. Preventive Services Task Force recommendation statement. Ann Intern Med. 2008;148(11):846-854.

29. Hoerger TJ, Hicks KA, Sorensen SW, et al. Cost-effectiveness of screening for pre-diabetes among overweight and obese U.S. adults. Diabetes Care. 2007:30(11):2874-2879. 